\title{
Research Paper: Immediate Effects of Different Screen Sizes on Visual Fatigue in Video Display Terminal Users
}

\author{
Sirinthip Pakdee ${ }^{1}$ (D) Praphatson Sengsoon $^{1^{*}}$ (D)
}

1. Department of Physical Therapy, School of Allied Health Sciences, Walailak University, Nakhon Si Thammarat Province, Thailand.

\begin{tabular}{|c|c|}
\hline $\begin{array}{l}\text { Use your device to scan } \\
\text { and read the article online }\end{array}$ & \\
\hline 口畐乐回 & Citation: Pakdee S, Sengsoon P. Immediate Effects of Different Screen Sizes on Visual Fatigue in Video Display Terminal \\
\hline 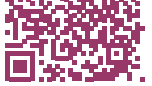 & doi'http://dx.doi.org/10.32598/irj.19.2.1108.2 \\
\hline
\end{tabular}

Article info:

Received: 01 Apr 2021

Accepted: 26 Apr 2021

Available Online: 01 June 2021

Keywords:

Screen size, Visual fatigue, Critical flicker frequency, Dry eyes, Video display terminal

\section{ABSTRACT}

Objectives: Computer usage has rapidly grown. This is because it helps to resolve problems, i.e., encountered in daily life by individuals. Various monitor screens that have been developed affect the user's eyes. Screen size is one of the relevant impacts. Thus, this study compared the immediate effects of two computer screen sizes on visual fatigue in Video Display Terminal (VDT) users.

Methods: Twenty female VDT users participated in this study. Using a randomized block design for the study, the study participants randomly drew a ballot to determine the order of using an 18.5-inch and 23-inch computer screen size. The research participants were assessed by a visual fatigue score, critical flicker frequency, and dry eye score before and after using both computer screen sizes. They were tested in an ergonomic computer workstation for 2 hours. Besides, where they rested between each workstation for $\geq 30$ minutes or until presetting no eye fatigue symptoms. The relevant data were compared between before and after using the computers and between the two different screen sizes.

Results: The collected results suggested no significant difference in the visual fatigue score, critical flicker frequency, and dry eye score between using either computer screen sizes $(\mathrm{P}>0.05)$. However, there were significant differences in the visual fatigue score, critical flicker frequency, and dry eye score between before and after computer screen usage $(\mathrm{P}<0.05)$.

Discussion: Using both computer screen sizes resulted in increased visual fatigue, reduced critical flicker frequency, and increased dry eyes. The present study results can provide information in determining how to reduce risk factors and prevent visual fatigue from continuous computer use for a long time. 


\section{Highlights}

- Continuous computer use for 2 hours presents risk factors of visual fatigue.

- The 18.5-inch and 23-inch computer screen sizes indicated no different effects on visual fatigue in video display terminal users.

\section{Plain Language Summary}

Computers and their usage have rapidly and widely grown worldwide. However, using a computer may affect eye fatigue. This is because computer users are less active and tend to sit in the same position for a long time to focus on their work. Nowadays, computer monitors come in various sizes to provide the most effective one for users. Hence, this study aimed to compare the immediate effects of using two computer screen sizes on eye fatigue in computer users. Using a randomized block design, 20 female computer users randomly drew a ballot to determine the order of using an 18.5-inch and 23-inch computer screen size. The study participants were assessed a visual fatigue score, critical flicker frequency, and dry eye score before and after using both computer screen sizes. They were evaluated in an ergonomic computer workstation for 2 hours. Moreover, they rested between each workstation until there were no eye fatigue symptoms. The collected results suggested that using both computer screen sizes resulted in increased visual fatigue, reduced critical flicker frequency, and increased dry eyes. This information can be used to reduce risk factors and prevent visual fatigue from continuous computer use for a long time.

\section{Introduction}

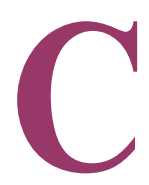

omputers are the product of modern technology to help facilitate comfort and improve work efficiency. Computers play an essential role in our daily life, where the rate of their use has rapidly increased [1] according to a survey conducted by the National Statistical Office from 20142018. It was found that there are 17.9 million computer users out of a population consisting of 63.3 million individuals in Thailand [2]. Moreover, computer usage for 5 days per week was equal to $57.7 \%$; the duration of computer use for $8 \mathrm{~h} / \mathrm{d}$ was measured as $32.6 \%$ [3]. However, using a computer inevitably affects the biopsychological system of the user. This is because computer users are less active and tend to sit in the same position for a long time to focus on their work. The most frequent problems among computer users consist of visual problems (75\%90\%), including Computer Vision Syndrome (CVS) [4, 5] and other visual defects, such as decreased visual acuity, myopia, etc. [1, 6]; followed by musculoskeletal issues, with $22 \%$ involving pain in the neck, shoulder, wrist, and back regions $[5,7]$. The least common problems consist of psychological conditions, including stress and depression [4].

Eye fatigue is among the most significant problems encountered by computer users [8]. A study in Thailand found that the prevalence of eye fatigue among comput- er users equaled $76.6 \%-96.4 \%$ [5]; it was caused by the continuous and prolonged use of the eyes on a computer screen. Additionally, computer usage for $>2$ hours [1] presents a 50\%-90\% risk of developing eye fatigue [9], i.e., mainly caused by contracted eye muscles. The eyes shrink while staring at a computer screen for an extended time [5]. Computer use has also decreased the blink rate, increasing the evaporation rate of tears, i.e., the cause of eye dryness. This effect is a symptom of eye fatigue, which leads to declined visual efficiency [10].

Eye fatigue can be assessed by various methods, such as an asthenopia questionnaire, a dry eye questionnaire [11, 12], and a critical flicker frequency meter. Furthermore, the relationship between various factors that affect eye fatigue is also impactful in testing visual acuity $[5,13]$.

Computers are evolving through various types, screen sizes, and graphics to accommodate the needs of users [14], where computer monitors have the greatest effect on users. In Thailand, it was found that the most commonly used screen size was 18.5 inches, followed by 21.5 inches, and 23 inches [15]; a survey of computer use at Walailak University found that the most common computer screen size was 18.5 inches, followed by 23 inches.

A study investigated the relationship between different computer screen sizes and the appropriate computer screen and keyboard placement. The achieved results found that increased screen size enhances the viewing 
distance and decreases the screen height [14]. This study also suggested that increasing the distance between the eyes and the computer screen resulted in a significant reduction in dry eyes, blurred vision, and headaches [13]. Additionally, previous studies demonstrated that decreasing the distance between the eyes and the computer screen contributed to visual fatigue, with a significant decrease in the frequency of flashing light [5]. Computers are developed in various sizes and screen styles [13, 15]. However, studies comparing the effects of different computer screen sizes on eye fatigue are scarce.

Thus, this study aimed to compare the immediate effects of using two computer screen sizes on eye fatigue in computer users among the general population at Walailak University. We aimed to gather information for developing suitable screen size guidelines and eye fatigue prevention.

\section{Methods}

This was a descriptive and cross-sectional study. This study included data collection and analysis for visual fatigue while using small and large screen sizes.

Twenty subjects were calculated from a pilot study (effect size $=0.28$, power $=0.8, \& \mathrm{P}=0.05$ ). The research subjects were computer users selected from Walailak University according to the selection criteria. The inclusion criteria consisted of female gender, 18-25 years of age, Body Mass Index (BMI) in the range of 18.5-22.9 $\mathrm{kg} / \mathrm{m} 2$, continuous computer use of at least 2 hours, computer use of at least 5 days per week, an experience of computer usage for at least 1 year, right-hand dominance, immediate typing speed in the range of 2035 words per minute, and visual acuity at 20/20 level. Subjects were excluded if they had eye disorders, such as color blindness, cataracts, nearsightedness, farsightedness, and astigmatism $[12,16]$. Moreover, the subjects with musculoskeletal symptoms and disorders that affect computer use (e.g., neck, back, or shoulder pain), neurological symptoms that affect computer use (e.g., Vertebrobasilar Insufficiency [VBI] or vestibular disease, etc.), drank alcoholic and caffeinated beverages within 24 hours prior to the test [8], took medication that may affect the test (e.g., pain relief drugs, muscle relaxants, $\&$ drugs that affect the nervous system) within 24 hours before testing, and those with psychological problems with a score $>25$ according to the Department of Mental Health assessment form (SPST-20) [17] were excluded for this study.
This study was approved by the Human Research Ethics Committee, Walailak University (Code: WUEC-19205-01). The study subjects were informed about the research. They were also required to provide signed informed consent forms to participate in this research before data collection.

The study subjects used both an 18.5-inch and a 23inch computer screen. Eligible subjects were randomly assigned in a randomized block order to determine the computer screen size usage order for the test. Afterward, the researcher arranged the suitable workstation, computer screen resolution, and computer screen brightness for the study subjects using ergonomic guidelines. The light intensity in the room was controlled to be between 450-500 lux. Before the test, the subjects were provided 5 minutes to become familiar with the computers. Before and after testing with each computer screen size, the research subjects were required to complete the asthenopia questionnaire; it consisted of 10 questions to assess eye fatigue. The 14-item dry eye questionnaire was provided to assess eye dryness in the study participants. Both questionnaires are reliable $[11,12]$. Eye fatigue was also assessed with a critical flicker frequency machine, i.e., valid and highly reliable. It was conducted by having subjects look at a flashing color spectrum with an everincreasing frequency. If the subjects were unable to see the blinking band, they were instructed to press the stop button to record the Critical Flicker Frequency (CFF) value in cycles per second (cycle per second or Hertz). Each measurement lasted approximately 20 seconds.

For the testing process, the study subjects were required to sit and type articles using the computer for 2 hours on the first computer screen size. Besides, the order of screen size usage was randomized. The research subjects were given at least 30 minutes of rest or until there was no visual fatigue before the second computer screen size test was performed. Statistical data analysis was performed using SPSS at the significance level of $\mathrm{P}<0.05$. The selected statistics were as follows:

Comparing visual fatigue differences before and after using both computer screen sizes (18.5-inch \& 23-inch) by two-way mixed Analysis of Variance (ANOVA) and the Bonferroni test.

Comparing the CFF before and after using both computer screen sizes by the Shapiro-Wilk test to determine the data distribution. The collected data presented a normal distribution; thus, two-way mixed ANOVA and the Bonferroni test were employed. 
Comparing dry eye differences before and after using both computer screen sizes by two-way mixed ANOVA and the Bonferroni test.

\section{Results}

This study compared the immediate effects of using two different computer screen sizes on eye fatigue. This measure was assessed by analyzing the results of the level of visual fatigue, CFF, and dry eyes in female computer users at Walailak University.

The general characteristics of the 20 female participants were as follows: Mean \pm SD age: $22.00 \pm 1.26$ years, weight: $54.45 \pm 6.26 \mathrm{~kg}$, height: $159.95 \pm 7.03$ $\mathrm{cm}$, BMI: $21.24 \pm 1.39 \mathrm{~kg} / \mathrm{m}^{2}$, the duration of computer use: $2.75 \pm 0.85$ hours, and computer use experience: $9.48 \pm 2.02$ years (Table 1 ).

There was no significant difference in visual fatigue between using the 18.5-inch and 23-inch computer screens $(\mathrm{P}=0.806)$. The Mean $\pm \mathrm{SD}$ visual fatigue score for the 18.5-inch and 23-inch screen sizes was $18.45 \pm 6.21$ and $18.90 \pm 7.71$, respectively. There was a significant difference in visual fatigue between before and after using the 18.5-inch and 23-inch computer screens, $(\mathrm{P}<0.001)$ (Figure 1).

The statistical analysis of the difference between CFF when using the 18.5-inch and 23-inch computer screens indicated no significant difference $(\mathrm{P}=0.913)$. The Mean \pm SD value of the CFF for the 18.5-inch and 23 -inch screen sizes was $39.18 \pm 2.35$ and $39.09 \pm 2.52$, respectively. There was a statistically significant difference in CFF before and after using the 18.5-inch and 23-inch computer screens $(\mathrm{P}<0.05)$ (Figure 2).

There was no significant difference in dry eyes between using the 18.5-inch and 23-inch computer screens $(\mathrm{P}=0.737)$. The Mean $\pm \mathrm{SD}$ dry eye score for the 18.5 -inch and 23-inch screen sizes was 24.9 \pm 8.11 and $24.1 \pm 6.81$, respectively. There was a significant difference in dry eyes before and after using the 18.5-inch and 23-inch computer screens $(\mathrm{P}<0.05 \& \mathrm{P}<0.001)$ (Figure 3 ).

\section{Discussion}

The current study results suggested that continuous computer use for a long time affected visual fatigue when using small and large computer screens. The collected results can provide evidence for the effects of computer usage and can be used as a basis for finding approaches to reduce risk and prevent visual fatigue.
We also compared visual fatigue before and after using the 18.5-inch and 23-inch computer screen sizes; accordingly, there was a significant difference due to the continuous use of the computer for a long time causing the risk of visual fatigue. This condition is caused by eye muscles contracting over a longer period or more frequently [1] Moreover, mechanisms concerning visual abnormalities are involved; the eyes are used to look close-up, including the need to read texts from computer screens and documents [18]. Therefore, the eyes must adjust to the sharpness of the image to be as clear as possible. In this case, the crystalline lens act to adjust for the light that hits the retina together with the ciliary muscle contraction to make the lens work according to the adjustment of the image sharpness. Furthermore, the medial rectus muscle is contracted so that both eyes are directed together or at convergence. Therefore, when using the computer for a long time, this induces the ability to make accommodations and decreases convergence, leading to eye fatigue $[6,18]$. Moreover, visual fatigue may occur from the malfunction of the eye surface; subsequently, it generates dry eyes resulting in tears at the eye lens surface and causing problems with the lens of the eye [6].

Eye fatigue can also be caused by the interference of the light from the computer and light from other sources, i.e., combined to produce a glare on the computer screen [18]. The extent of light intensity in the workstation room for this study was controlled to range between 450 500 lux. This value is the amount of light that presents the least impact on the eyes. If the light intensity is $>500$ lux, it may cause excessive reflection of the light from the computer screen to the eyes of the computer user Accordingly, it requires a greater effort for the eyes to focus and produces reflections that cause discomfort, fatigue, and a decrease in visual efficiency of the eyes $[3$, $8]$. However, if the light intensity is $<300$ lux, there is too little light. This causes the eye muscles to work harder to force the iris to open from the gaze. This is because vision is unclear, resulting in eye fatigue as well [5].

Additionally, computer monitors have a refresh rate or vertical frequency, i.e., another factor that affects eye fatigue. This study was set at a rate of 75 BPM $(75 \mathrm{~Hz})$ as an appropriate value, which preserves most eyes $[1,5]$. Another factor that impacts the eyes is the resolution of the computer screen, i.e., displayed as an image. It is the part that affects the adjustment of the eyes by displaying a computer image that consists of a set of pixels. When using the computer for a long time, the eyes must adjust to receiving the displayed image sharply causing the eyes to lag after using the computer [5]. 
Table 1. General characteristics of the study participants $(\mathrm{N}=20)$

\begin{tabular}{cccc}
\hline General Characteristics & Mean \pm SD & Max. & Min. \\
\hline Age $(\mathrm{y})$ & $22.00 \pm 1.26$ & 24 & 19 \\
\hline Weight $(\mathrm{kg})$ & $54.45 \pm 6.26$ & 65 & 43 \\
\hline Height $(\mathrm{cm})$ & $159.95 \pm 7.03$ & 171 & 150 \\
\hline Body mass index $\left(\mathrm{kg} / \mathrm{m}^{2}\right)$ & $21.24 \pm 1.39$ & 22.83 & 2 \\
\hline Computer usage time $(\mathrm{h})$ & $2.75 \pm 0.85$ & 4 & 59 \\
\hline Computer experience $(\mathrm{y})$ & $9.48 \pm 2.02$ & 13 & 5 \\
\hline
\end{tabular}

The present study results suggested no significant difference in visual fatigue scores between the 18.5-inch and 23-inch computer screens. This may be because using both screen sizes presents a similar gaze; viewing the angles of the computer screen, keyboard, and documents, i.e., not over the same visual field. With the same working duration, there was no significant difference in the effect of using the explored computer screens on the eyes. This finding was consistent with a previous study that determined the effects of different angles of the eyesight and computer screen concerning visual acuity and blink rate. There was no significant difference in visual acuity and blink rate [19]. However, a study compared the differences in using 3 e-reader LCD screen sizes (6inch, 8.1-inch, \& 9.7-inch) on eye fatigue. They found that the 6-inch screen size, i.e., the smallest screen size, could cause eye fatigue significantly higher than the larger screen sizes. This effect is because the characters displayed on the screen were also smaller and required more gaze to focus on [20].

In addition, $\mathrm{Wu}$ et al. conducted a comparative study respecting screen size differences in 3 screen types, as follows: Personal Digital Assistant (PDA), LCD e-reader, and notebook. The PDA, i.e., the smallest screen, resulted in a significant increase in eye fatigue [19]. This resulting study was inconsistent with those of our research, which may be due to the PDA screen size being very small. Compared to this study, it was found that the screens were in 3 different sizes; accordingly, eye fatigue may be evident when the screen size is very small. Moreover, the eyes must focus more and the eye muscles have to work harder for smaller screen size. Moreover, our study compared the screen sizes of 18.5 inches and 23 inches, i.e., similar in size with a possibility that the 18.5inch size was not small enough to make a difference.

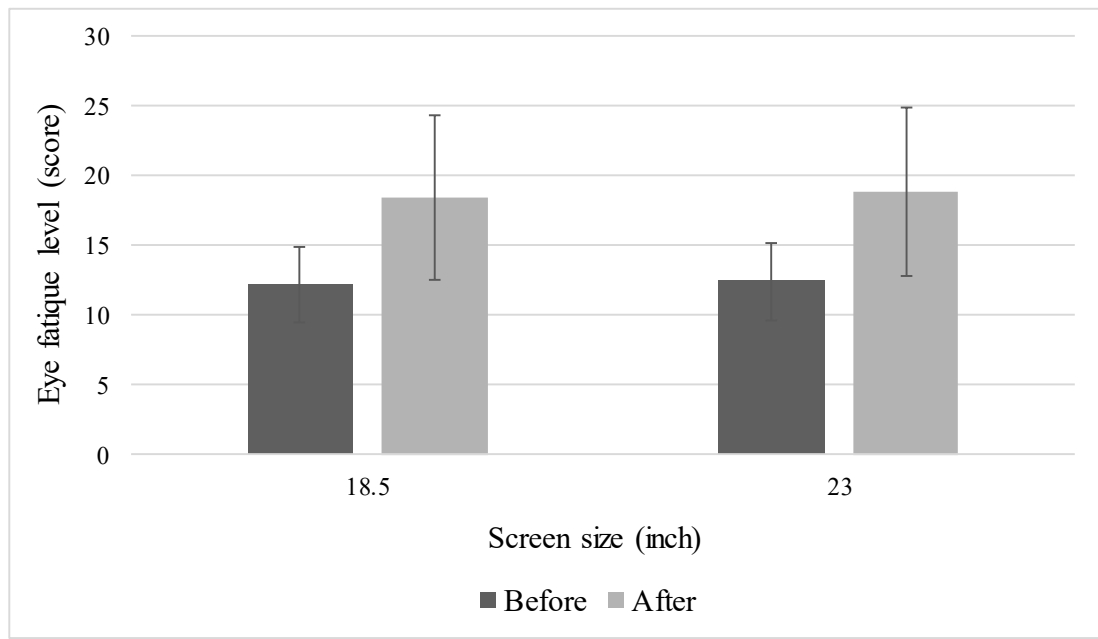

Iranian Rehabilitation Journa

Figure 1. Comparing the differences in visual fatigue when using 18.5-inch and 23-inch computer screens as well as before and after using both computer screen sizes

* $\mathrm{P}<0.05 ;{ }^{* *} \mathrm{P}<0.001$ 


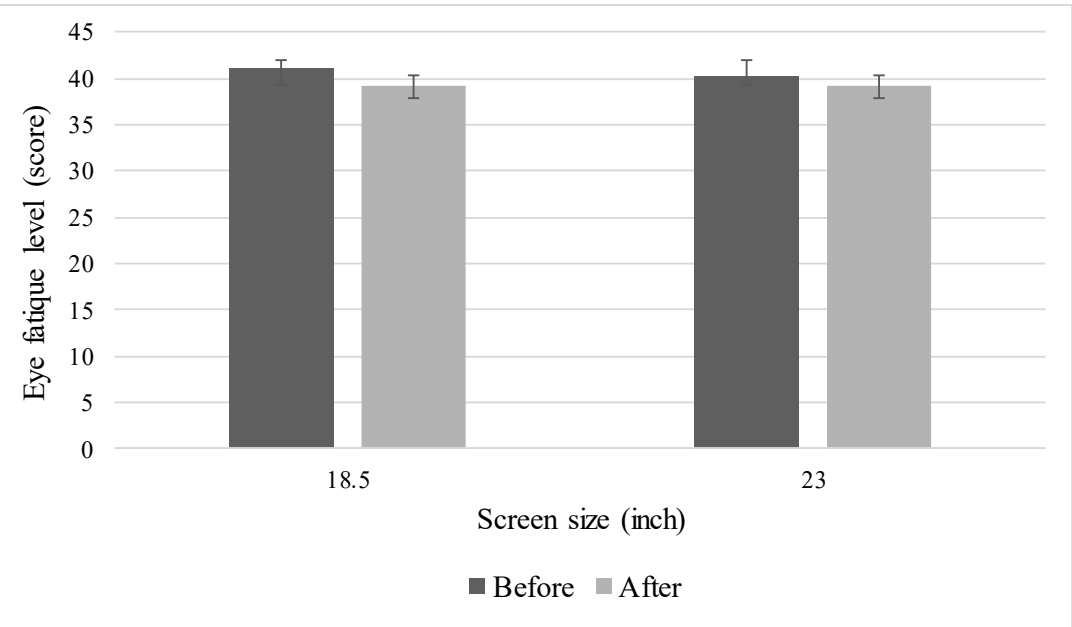

Iranian Rehabilitation Journal

Figure 2. Comparing the differences in CFF when using 18.5-inch and 23-inch computer screens and before and after using both computer screen sizes

${ }^{*} \mathrm{P}<0.05 ; * * \mathrm{P}<0.001$

There was a significant difference in the CFF between before and after using the 18.5-inch and 23-inch computer screen sizes. The obtained data indicated the occurrence of visual fatigue after computer use. This is because using the computer screen was at a close-up view for the eyes without changing the viewing distance. Therefore, the eyes had to adjust to the contrast of the image [6]. CFF is a well-accepted measure of visual fatigue [21]. Additionally, CFF can indicate a mental workload, i.e., correlated with task demand, effort, and performance [22].

CFF presents the collaboration between the eyes and the brain, i.e., characterized by the frequency that cannot be distinguished from constant light and non-flashing light. Decreased CFF is caused by a reduction in the functioning of the retina $[5,23]$. It is responsible for the visual acuity of the eye; it transforms into a nerve current sent to the nerve terminal and the second cranial nerve (optic nerve). It is crucial to observe whether using the computer screen is at a close-up view and has been looked at for a long time. The eyes have to adjust to the contrast of the image. Crystalline lenses have to work by modulating the incident light on the retina. This condition may result in visual fatigue, as the eyes perceive CFF is reduced. This causes the CFF to be reduced by $<30$ cycles per second. Moreover, there is no fatigue in the eyes, which can perceive high critical flicker frequency. As a result, when the CFF is $\geq 40$ cycles per second, this results in a negative change in the CFF; accordingly, it is expressed as symptoms, such as eye pain, itchy eyes, or a feeling of heaviness in the eyes.
The current research results were consistent with those of previous studies. It was found that after the study subjects used the computer screen for a while, this resulted in a decrease in CFF. Besides, there was an increase in visual fatigue and dryness of the eyes based on the questionnaires [24]. The collected results revealed that after 2 hours of continuous use of the computer screen, CFF was decreased. These data were consistent with those of Chi and Lin; they documented a significant increase of sensitivity in CFF that occurred following the increased period of computer screen usage. In other words, using computers for a long time decreases CFF; the longer the computer screen is used, the more rapidly and CFF decreases [25]. Moreover, the obtained results were consistent with those of a previous study. Similarly, they found that the factors affecting visual fatigue measured by the CFF included the duration of continuous computer usage for $>2$ hours [5]. Most studies found that using a computer for $>2$ hours causes visual fatigue that aggravates the duration of computer use.

Looking at a computer screen involves the visual mechanism that produces images by the refraction of light around the cornea and the lens; it can adjust the focal length to achieve clarity on the cornea (retina). Staring at characters on a computer screen causes the eyes to focus on the monitor. Accordingly, the eye muscles will automatically contract to see sharp images all the time. The eye muscles working hard for a long period results in visual fatigue. Subsequently, the CFF measurement is reduced [26]. Sheedy conducted a study on visual problems in computer users who used computers for a long period [27]. They found a decrease in image 


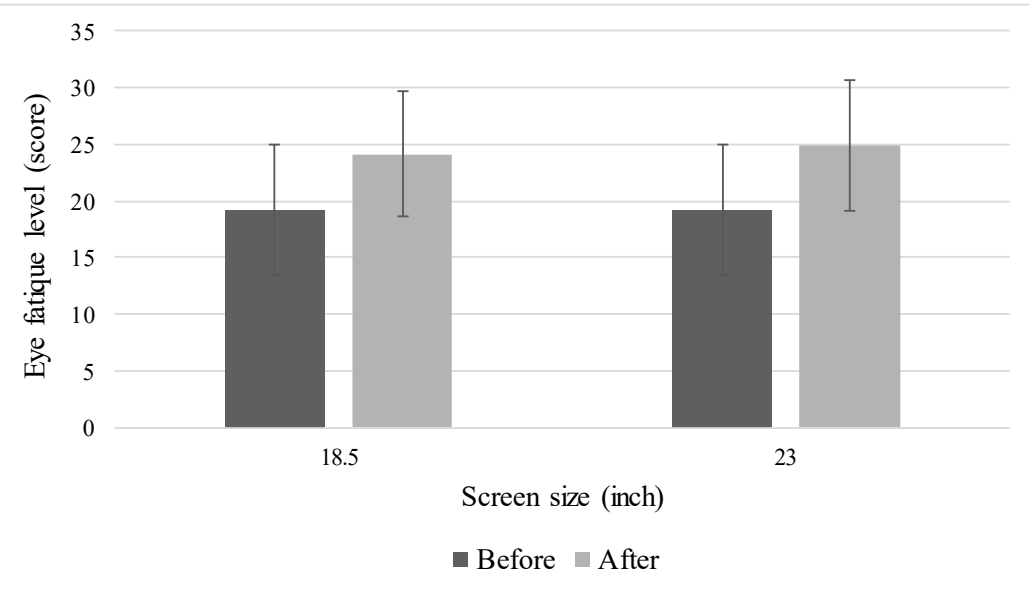

Iranian Rehabilitation Journal

Figure 3. Comparing the differences in dry eyes between using 18.5-inch and 23-inch computer screens as well as before and after using both computer screens sizes

* $\mathrm{P}<0.05 ; * * \mathrm{P}<0.001$

sharpness adjustment (accommodation) over time. This can be described as the need for the eyes to move and sharpen the image when looking at characters on the computer screen. As a result, the eyes need to focus more on the computer screen to be able to view it more clearly. Therefore, visual fatigue can occur, which increases over time when using a computer screen [27].

Additionally, the obtained data revealed a difference in CFF between using the 18.5-inch and 23-inch computer screens. However, there was no significant difference between the 18.5-inch and 23-inch screens, i.e., possibly due to the similar typing characteristics when looking at the computer screen, keyboard, and documents for both screens. Other factors might include the similarity in the amount of light on the computer screen, the displays of computer images, and the controls of the workstation.

The collected results suggested a significant difference in the dry eye scores between before and after using the 18.5-inch and 23-inch computer screen sizes. Due to using the computer for a long time, this required focusing and looking close-up at the computer screen continuously; consequently, it reduced eye movement. Moreover, this condition led to a decrease in the blink rate and an increase in the evaporation rate of tears; accordingly, it generated dry eyes, tear film anomalies, and malfunction of the eye surface [28]. Furthermore, the prolonged use of the computer causes the ciliary body, which controls tear production and the adjustment of the sharpness of each vision (focusing), to work continuously for a long time; subsequently, it results in poor vision and decreased ability to adjust the contrast of vision at each distance.
Moreover, previous studies reported that most computer users experience dry eyes. Two main causes have been found for dry eyes, including a reduction in the tear production from the lacrimal gland and a loss of more tears from the evaporation of tears. These factors are essential in computer screen use. Therefore, computer use can decrease the blink rate and lead to dry eyes, where the normal condition for the blink rate is 10-22 BPM [29].

The blink is caused by the orbicularis oculi muscle activity to stimulate the lacrimal gland for eye surface coating and eye moisturizing, which prevents dry eyes [29]. The eyes can be divided into 3layers, as follows: the outermost layer is the lipid layer that acts to prevent evaporation of tears; the middle layer is a layer of water (aqueous layer) that serves to protect against foreign matter as well as pathogens entering the eye; the innermost layer is the mucous layer (mucin layer) that serves to distribute tears evenly across the cornea. All 3 layers are critical to protect and help moisturize the eyes. If a particular layer has an abnormality, this can result in dry eyes [1].

Previous reports indicated that using a computer screen can reduce the blink rate to $7.6 \pm 6.7$ BPM. Thus, it causes tears to coat the eye surface less than usual and increases the evaporation rate of tears. This is the cause of dryness and the lack of moisture for the eyes. Tsubota and Nakamori conducted a study on 2 hours of computer usage respecting dry eyes. They concluded that the Mean \pm SD blink rate in a relaxed state was $22 \pm 9$ BPM; however, when using a computer, the same rate dropped to $7 \pm 7 \mathrm{BPM}$, causing dry eyes [30]. Using computers also has the potential for incomplete blinking when the upper and lower eyelids are not completely closed; subsequently, it causes the evapo- 
ration of tears, i.e., greater than normal, resulting in dry eyes as well. Besides, using a computer screen requires the use of the eyes in a horizontal manner (horizontal gaze) that makes the gap between the upper and lower eyelids wider than when reading in general. Therefore, the surface area of the eye is larger, increasing the evaporation rate of the tear film and causing dry eyes [30].

Additionally, this study demonstrated no significant difference in the dry eye scores between the 18.5-inch and 23-inch computer screens. This may be attributed to the similar use of both computer screen sizes, where subjects had to use their eyes to look at the computer screen, keyboard, and documents, alternating over time with the same job characteristics. In the use of both screen sizes, the eyes must gaze in the same way while performing the same work.

Two hours of computer use was a limitation in applying the study results to a population with 8-10 hours of computer use. Furthermore, the study participants in the study had normal vision, making it a limitation in applying the results of the study to a population with various levels of impaired vision. Finally, the study participants were females, restricting generalizing the obtained results to males.

According to the collected results, using a computer monitor (18.5-inch \& 23-inch) could cause visual fatigue, i.e., indicated by the results of the assessment on visual fatigue, critical flicker frequency, and dry eyes. Therefore, users can choose to use either computer screen size. However, users should be careful when using computers for a long time. Individuals who work with a computer for 2 hours can develop eye strain due to an abnormality where visual acuity adjustment is slower. This is caused by the eye muscles contracting over a longer period or more often. It may also be caused by a decrease in the blink rate, which generates the dryness of the eyes and leads to visual fatigue.

Recommendations for future studies include the following: exploring the long-term effects of visual fatigue when using both computer screen sizes (18.5-inch \& 23inch); examining the duration of the outstanding effects on visual fatigue when using both computer screen sizes; investigating visual fatigue when gazing at a different screen (e.g., tablet screen or smartphone screen), and determining other factors causing visual fatigue when using both computer screen sizes (e.g., the resolution of the computer screen, brightness of the computer screen, etc.).

\section{Conclusion}

We explored the immediate effects of using two different computer screen sizes on visual fatigue among 20 female computer users at Walailak University. We assessed the visual fatigue score, CFF, and dry eye score. There was no significant difference in the visual fatigue scores when using both computer screens; however, a significant difference was found before and after the use of the 18.5-inch and 23-inch computer screens. Besides, there was no significant difference in the CFF when using both computer screens; however, a significant difference was found before and after the use of the 18.5-inch and 23-inch computer screens. Moreover, there was no significant difference in the dry eye scores when using both computer screens; however, a statistically significant difference was found before and after the use of the 18.5-inch and 23-inch computer screens. Therefore, using both computer screen sizes could induce visual fatigue, decrease critical flicker frequency, and increase the dryness of the eyes with a similar effect.

\section{Ethical Considerations}

\section{Compliance with ethical guidelines}

The study was approved by the Human Research Ethics Committee of Walailak University and was performed per the Declaration of Helsinki. All participants signed written informed consent forms before the commencement of the study.

\section{Funding}

This study was granted by the Department of Physical Therapy, School of Allied Health Sciences, Walailak University, Nakhon Si Thammarat Province, Thailand.

\section{Authors' contributions}

Conceptualization: Praphatson Sengsoon; Methodology: Sirinthip Pakdee and Praphatson Sengsoon; Investigation: Sirinthip Pakdee; Writing - Original Draft: Sirinthip Pakdee; Writing - Review \& Editing: Praphatson Sengsoon; Funding Acquisition: Praphatson Sengsoon; Resources: Praphatson Sengsoon; Supervision: Praphatson Sengsoon and Sirinthip Pakdee.

\section{Conflict of interest}

There were no conflicts of interest to be declared. 


\section{References}

[1] Lavin W, Taptagaporn S, Khruakhorn S. Computer vision syndrome, CVS: One case report in children. Thammasat Medical Journal. 15(1):136-42. https://he02.tci-thaijo.org/index.php/tmj/article/view/33186

[2] Natioanal Statistical Office. Number of information and communication technology devices in household by region and province in 2019. Information and Communication Technology (ICT) Survey in Thailand. 2019; 1:1-54. https:/ / datareportal.com/reports/digital-2019-global-digital-overview

[3] PhonharnN RS, Boonkaew K, Seewirat A. [The physical symptoms that cccur from using computer of supporting staffs of Nakhonphanom University (Thai)]. Srinakharinwirot University. Journal of Science and Technology. 2014; 6(12) 26-38. http://ejournals.swu.ac.th/index.php/SWUJournal/ article/view/5017

[4] Gowrisankaran S, Sheedy JE. Computer vision syndrome: A review. Work. 2015; 52(2):303-14. [DOI:10.3233/WOR-152162]

[5] Sornboot J, Phakthongsuk P, Thangtrison S. [Prevalence of visual fatigue and its determinants among computer users in the Faculty of Medicine, Prince of Songkla University (Thai)]. Songklanagarind Medical Journal. 2009; 27(2):91-104. http:// smj.medicine.psu.ac.th/index.php/smj/article/view/289

[6] Chidnayee S, Chidnayee J, Kuariyakul A. Risk factors for computer visual syndrome and prevalence of vision problems in nursing students at Borommarajonani College of Nursing Uttaradit. Nursing Public Health and Education Journal. 2013; 1(15):13-22. http://web.bcnpy.ac.th/journal/images/ file/pdf/year16-no2-may-august-2558-ISBN0859-3949.pdf

[7] Pituksung A, Tanterothum J, Supharerx S, Waranukulsak O. The study of anxiety, stress and information required of the preoperative outpatients. Journal of Nursing Science. 2011; 4(1):3942. https://www2.si.mahidol.ac.th/division/nursing/sins/ attachments/article/33/sins-journal-2554-01_page35-42.pdf

[8] Habibi E, Rajabi H, Arbabi M. An examination of the relationship between visual fatigue symptoms with flicker value variations in video display terminal users. Iranian Journal of Health, Safety and Environment. 2015; 2(1):211-6. http:// ijhse.ir/index.php/IJHSE/article/view/71

[9] Thongmeekhaun T, Tiaw SS, Juntaveemuang V. [Computer syndrome: Imminent hazard that can be perceived and preventable (Thai)]. Nursing Journal of The Ministry of Public Health. 2017; 27:19-31. https://he02.tci-thaijo.org/index. $\mathrm{php/tnaph/article/view/112200}$

[10] Kozeis N. Impact of computer use on children's vision. Hippokratia. 2009; 13(4):230-1. [PMID] [PMCID]

[11] Toda I, Fujishima H, Tsubota K. Ocular fatigue is the major symptom of dry eye. Acta Ophthalmologica. 1993; 71(3):34752. [DOI:10.1111/j.1755-3768.1993.tb07146.x]

[12] Kim DJ, Lim CY, Gu N, Park CY. Visual fatigue induced by viewing a tablet computer with a high-resolution display. Korean Journal of Ophthalmolog. 2017; 31(5):388. [DOI:10.3341/ kjo.2013.27.5.388]

[13] Maeda E, Yoshikawa T, Hayashi N, Akai H, Hanaoka S, Sasaki H., et al. Radiology reading-caused fatigue and measurement of eye strain with critical flicker fusion frequency. Japanese Journal of Radiology. 2011; 29(7):483. [DOI:10.1007/ s11604-011-0585-7]
[14] Shin G, Hegde S. (2010). User-preferred position of computer displays: Effects of display size. Human Factors. 2010 52(5):574-85. [DOI:10.1177/0018720810380405] [PMID]

[15] Kemp S. The global state of digital [Internet]. 2019 [Updated January 31]. Global digital overview. Available from: https:// datareportal.com/reports/digital-2019-global-digital-overview

[16] Porcar E, Pons AM, Lorente A. Visual and ocular effects from the use of flat-panel displays. International Journal of Ophthalmology. 2016; 9(6):881-5. [PMID] [PMCID]

[17] Mahatnirunkul S, Phumpisarnchai W, Tarpunyar P. Suanprung stress test. Bull Suanprung. 1997; 13:1-20. http:/ /www. cumentalhealth.com/index.php?lay $=$ show \&ac $=$ article\& $\mathrm{Id}=539909295$

[18] Photihung P, Srisopa P, Tassanatanachai A. [Risk factors of computer vision syndromes among instructors, Burapha University (Thai)]. Journal of Nursing and Education. 2016; 9(2):104-19. file:///C:/Users/b.fallahi/Downloads/57027Article\%20Text-141587-1-10-20160630.pdf

[19] Wu HC, Lee CL, Lin CT. Ergonomic evaluation of three popular Chinese e-book displays for prolonged reading. International Journal of Industrial Ergonomics. 2007; 37(910):761-70. [DOI:10.1016/j.ergon.2007.06.003]

[20] Turville KL, Psihogios JP, Ulmer T R, Mirka GA. The effects of video display terminal height on the operator: A comparison of the 15 and 40 recommendations. Applied Ergonomics. 1998; 29(4):239-46. [DOI:10.1016/S0003-6870(97)00048-3]

[21] Eisen-Enosh A, Farah N, Burgansky-Eliash Z, Polat U, Mandel Y. Evaluation of critical flicker-fusion frequency measurement methods for the investigation of visual temporal resolution. Scientific Reports. 2017; 7(1):1-9. [DOI:10.1038/ s41598-017-15034-z]

[22] De Waard D. The measurement of drivers' mental workload [PhD dissertation]. Netherland: University of Groningen ;1996. file:/ //C:/Users/b.fallahi/Downloads/1\%20(1).pdf

[23] Sheppard AL, Wolffsohn JS. Digital eye strain: Prevalence, measurement and amelioration. BMJ Open Ophthalmology. 2018; 3:e000146. [DOI:10.1136/bmjophth-2018-000146]

[24] Lin JB, Gerratt BW, Bassi C J, Apte R S. Short-wavelength light-blocking eyeglasses attenuate symptoms of eye fatigue. Investigative Ophthalmology \& Visual Science. 2017; 58(1):442-7. [DOI:10.1167/iovs.16-20663]

[25] Chi CF, Lin FT. A comparison of seven visual fatigue assessment techniques in three data-acquisition VDT tasks. Human Factors. 1998; 40(4):577-90. [DOI:10.1518/001872098779649247]

[26] Richter HO, Crenshaw AG, Lyskov E. Accommodation-vergence performance after low levels of oculomotor load. SJWEH Supplements. 2007; 3:60-7. https:// www.airitilibrary.com/Publication/alDetailedMesh?doc id=17959926-200701-201011230066-201011230066-60-67

[27] Sheedy JE. Vision problems at video display terminals: A survey of optometrists. Journal of the American Optometric Association. 1992; 63(10):687-92. [PMID]

[28] Lemp MA. Report of the National Eye Institute/Industry workshop on clinical trials in dry eyes. The CLAO Journal. 1995; 21(4):221-32. [PMID] 
[29] French K, Veys J. In the blink of an eye. Optometry Today. 2007; 47(18):40-3. https://www.jnjvisioncare.co.uk/sites/ default/files/public/uk/tvci/in_the_blink_of_an_eye_ot_ tvci_version.pdf

[30] Tsubota K, Nakamori K. Dry eyes and video display terminals. The New England Journal of Medicine. 1993; 328:584. [DOI:10.1056/NEJM199302253280817] 